\title{
Gestión ambiental en el Ejército Nacional de Colombia: oportunidades y perspectivas desde la economía circular ${ }^{1}$
}

\author{
Bart Van Hoof ${ }^{2}$ \\ Universidad de los Andes \\ Manuela Alejandra Parra Escobar ${ }^{3}$ \\ Universidad de los Andes
}

\section{Resumen}

El sistema lineal actual no solo ha aumentado la demanda de recursos naturales no renovables, es decir, aquellos que existen en una cantidad específica y limitada, hasta llegar a un punto de riesgo de escasez, sino que las malas prácticas que este sistema conlleva y la poca educación en el tema ha llevado a un aumento insostenible en los residuos. Todas estas prácticas han generado consecuencias evidenciables a nivel global, por lo que desde hace algunos ańos se ha empezado a idear e implementar este nuevo sistema circular, el cual limita tanto la extracción de materia prima como la producción de residuos con el objetivo de mantener constante el valor de los productos, materiales y recursos en el tiempo. La transformación hacia un sistema circular es un desarrollo que se debe llevar a cabo no solo en los procesos y elementos principales de las cadenas de suministro, sino también en las organizaciones e instituciones, de manera que se articule una red sostenible a nivel nacional. En ese orden de ideas, el presente trabajo expone una revisión teórica en torno a los impactos del sistema lineal principios y características bajo los que se estructura la economía circular, casos exitosos y modelos de estrategias tanto a nivel

1 Este capítulo hace parte de los resultados de la experiencia de los autores en las áreas de gestión cultura del reciclaje y otros temas ambientales ejecutados en la Universidad de los Andes. Los puntos de vista y los resultados de este capítulo pertenecen al autor y no reflejan necesariamente los de las instituciones participantes.

$2 \mathrm{PhD}$ en Ecología Industrial (Universidad Erasmo de Róterdam). Magíster en Ingeniería Industrial (Universidad de los Andes). Ingeniero Industrial (Universidad Hogeschool Eindhoven). Profesor asociado de la Facultad de Administración de la Universidad de los Andes. Director del área de sostenibilidad de la misma Facultad. Áreas de investigación en Gerencia Ambiental Empresarial y Ecología Industrial. Gerente de Desempeño Ambiental de Ecopetrol. Desarrollo de políticas públicas para el Ministerio de Ambiente y Desarrollo Territorial y Ministerio de Comercio e Industria. Consultor de Corporaciones Autónomas Regionales. Asesor principal de la Estrategia Nacional de Economía Circular del Gobierno Colombiano. Orcid:https://orcid.org/0000-0002-16186941 - Contacto:bvan@uniandes.edu.co

3 Ingeniera Ambiental (Universidad de los Andes). Estudiante Maestría en Gerencia y Práctica de Desarrollo (Universidad de los Andes). Asistente de investigación en temas de sostenibilidad, líder de proyectos encaminados a la cultura del reciclaje y otros temas ambientales. Orcid: https://orcid.org/0000-0002-0794-0842 Contacto:ma.parra@uniandes.edu.co 
comercial como a nivel de Fuerzas Militares. Todo ello, con el objeto de realizar un análisis de casos para la posible puesta en marcha de estrategias en el Ejército Nacional de Colombia, como, por ejemplo, la implementación de educación en desarrollo sostenible desde la escuela militar, la organización dentro de las instalaciones y la cohesión con otros actores para lograr investigación alrededor del tema.

Palabras clave: economía circular, cierre de ciclos, estrategia, desarrollo sostenible.

\section{Introducción}

La economía mundial actual maneja un sistema lineal, consecuencia de la industrialización y causa del crecimiento económico. De manera general, este se basa en 'tomar, hacer, usar y disponer' de los recursos con los que cuenta el planeta. Sin embargo, bajo este sistema los materiales y la energía no son aprovechados de una manera sostenible y la cadena de suministro presenta falencias en cada una de sus etapas: las fuentes primarias son sobreexplotadas y contaminadas; la producción de bienes y servicios funciona bajo técnicas y metodologías poco conscientes; las pérdidas en la distribución y venta de bienes y servicios son bastante notorias; los usuarios se ven cada vez más influenciados por el consumismo; y, finalmente, la tierra se convierte en un sumidero de residuos y contaminación.

Como consecuencia a estas falencias, se observan de manera cada vez más recurrente pérdidas económicas, incremento en la volatilidad de precios, riesgos de suministro, deterioro de los sistemas naturales, superación de la capacidad de carga de los rellenos sanitarios, entre otros (Ellen MacArthur Foundation, 2015). Un reflejo de estas consecuencias es el 'Día del Sobregiro de la Tierra' o 'Día de la Deuda Ecológica', fecha que establece el momento en el cual la humanidad ha utilizado todos los recursos biológicos que la Tierra puede renovar durante todo el año. Actualmente, se habla de un sobreuso del $60 \%$ de los recursos renovables, lo que se traduce en que la humanidad está usando 1.6 tierras para su sustento (Global Footprint Network \& Earth Overshoot Day, 2020). Esta cifra manifiesta una necesidad de cambio, puesto que su aumento paulatino desde la década de los setenta deja en evidencia los efectos del sistema económico actual, donde la necesidad de suplir a un mundo en sobrepoblación está acabando por agotar nuestras fuentes primarias.

Con base en estas consecuencias, y a la necesidad de proyectar un sistema económico que no agote las fuentes primarias del planeta, surge la economía circular, con un concepto fundamentado en la restauración y regeneración, distinguiendo los ciclos técnicos de los biológicos para generar mayor eficiencia. En esta 
economía se limita tanto la extracción de materia prima como la producción de residuos, con el objetivo de mantener constante el valor de los productos, materiales y recursos en el tiempo. Para garantizar una implementación y ejecución adecuados, esta economía se basa en tres principios: el primero consiste en preservar y mejorar el capital natural, controlando reservas finitas y equilibrando los flujos de recursos renovables; el segundo consiste en optimizar los rendimientos de los recursos distribuyendo productos, componentes y materias con su utilidad máxima en todo momento; finalmente, el tercer principio se centra en promover la eficacia de los sistemas detectando y eliminando del diseño los factores externos negativos (Ellen MacArthur Foundation, 2015).

De igual manera, la economía circular se concibe como un manifiesto de los sistemas naturales, en donde los residuos son fuente primaria en diferentes ciclos, la biodiversidad es generadora de resiliencia, la energía proviene de fuentes renovables y la clave de todo flujo efectivo se basa en pensar en sistemas donde confluyan personas, lugares e ideas para el desarrollo. De acuerdo con lo anterior, una implementación de este sistema no solo implicaría beneficios ambientales necesarios, sino un ahorro de billones en diferentes tipos de industrias, esto, debido a que gracias al cierre de ciclos la economía se expone menos a fluctuaciones en los precios de materiales, y la curva de costos, ahora aplanada, supone un uso más eficiente de recursos en términos de valor y volumen (Sariatli, 2017).

Pensando en las oportunidades que ofrece la economía circular, surge la necesidad de realizar casos de estudio, tanto para los diferentes actores de la cadena de suministro como para las instituciones sociales y del Estado que puedan acceder a las oportunidades que conlleva esta economía, en donde se identifiquen fortalezas y fallas en las que se pueda incurrir, resaltando los beneficios ambientales, sociales y económicos que una implementación de este sistema podría traer.

Con esto en mente, para el presente capítulo, se propone un estudio enfocado en el Ejército Nacional de Colombia, en donde, a través de un análisis teórico y metodológico, se comprenda el contexto ambiental, social y económico que se maneja en este tipo de instituciones, para así lograr una articulación de iniciativas que ahonden en la economía circular y sus necesidades de implementación, con base en su relación con los diferentes actores de la cadena de suministro.

Este análisis se realizará bajo el marco del 'Programa de Gestión Ambiental' que se viene implementando en el Ejército Nacional de Colombia, y con el objetivo de promover y fortalecer la investigación desde las diferentes escuelas de formación que hacen parte de las Fuerzas Militares (en adelante FF. MM.), en esta área del conocimiento, complementando así la visión de la Institución de ser "la fuerza de 
acción decisiva de la Nación, con capacidad de conducir operaciones autónomas, conjuntas, coordinadas y combinadas, en forma simultánea en dos teatros de operaciones, uno externo y/o uno interno" (Ejército Nacional de Colombia, s. p.).

\section{Marco teórico}

¿A cuántos le va bien cuando a la economia le va bien? ¿A cuántos desarrolla el desarrollo? EDUARDO GaLEANO, El libro de los abrazos

El último siglo ha traído consigo un avance tecnológico y un desarrollo industrial sin precedentes, sin embargo, como en todo sistema, se está llegando a un punto de quiebre en donde las exigencias globales no se ajustan a la capacidad de respuesta del planeta y sus recursos. A continuación, se presenta el alcance al que se ha llegado bajo el sistema económico actual y sus respectivas consecuencias.

\section{Sistema lineal}

Con el uso de la razón viene implícita la necesidad de desarrollo, sin embargo, por más impropio que parezca, esta necesidad parece no estar definida bajo los alcances de la madre tierra, sino bajo las exigencias de un hombre egoísta que apela a un sistema sin límites para abastecer su frenético crecimiento. Con estas palabras se podría describir brevemente el sistema económico que rige al mundo actualmente, un sistema lineal producto de dos revoluciones industriales que cambiaron la historia al introducir la mecanización, nuevos sistemas de obtención de energía, nuevos materiales y tecnología que aumentarían la eficiencia, cantidad, variedad y velocidad de producción (Andrews, 2015).

De esta manera, para entender estos avances y cómo se prevé la llegada a un punto de quiebre, es necesario comprender los principios bajo los que funciona este sistema: tomar, hacer, usar y disponer. Bajo estos cuatro términos se entiende que, para satisfacer las necesidades a una población en aumento acelerado, se realiza una extracción desmesurada de recursos naturales, estos recursos entran a una cadena de suministro enfocada en la producción masiva, producto de la necesidad de suplir a una clase media creciente (Bonciu, 2014), y que orienta al consumidor a obtener estos productos y servicios de manera más constante, ya sea por influencia de las tendencias del momento, por su baja calidad o porque ya no suplen las necesidades cambiantes (Lacy \& Rutqvist, 2016). Todo lo anterior conlleva a un aumento 
desmesurado de residuos y contaminación, lo que se puede evidenciar en lo reportado para el año 2016 por el Banco Mundial (ver figura 1), en donde se describe cómo el mundo genera aproximadamente 2.01 billones de toneladas de residuos sólidos anuales.

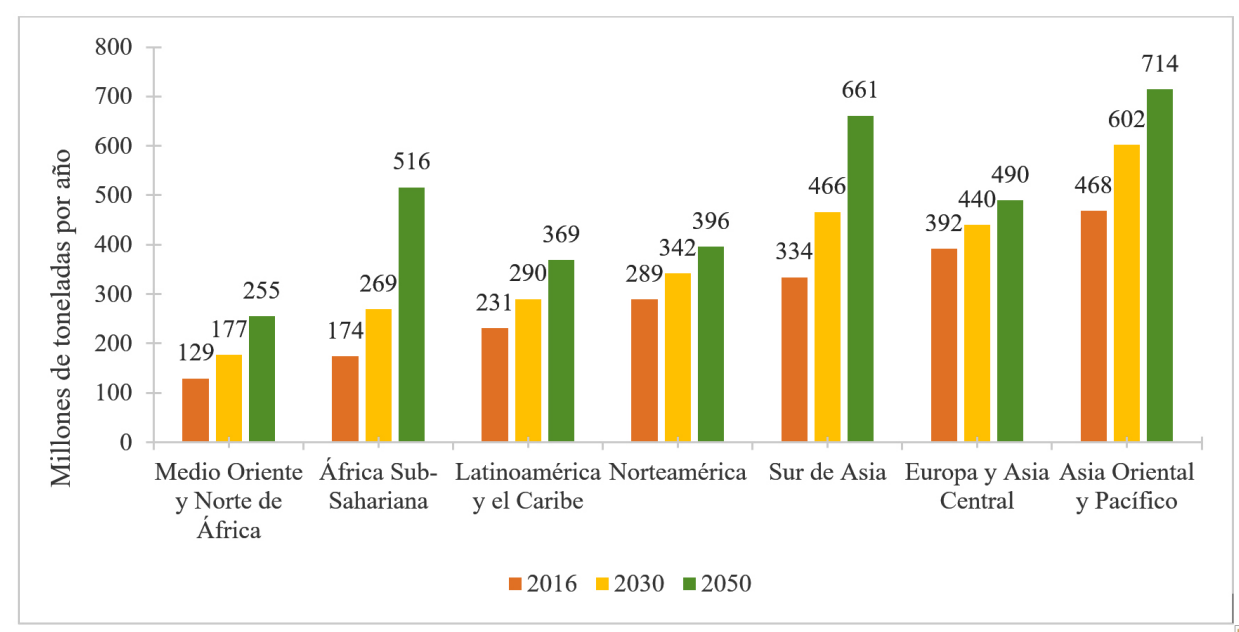

Figura 1. Generación de residuos por región (millones de toneladas) Fuente: adaptado de Banco Mundial (2016)

Adicionalmente, cómo se observa en la figura 1, las proyecciones para el 2050 no son nada alentadoras, con un aumento de aproximadamente $70 \%$ en la generación de residuos sólidos. De esta manera, aunque este sistema ha llevado a un importante crecimiento económico y ha generado múltiples avances en beneficio de la humanidad, es evidente que se trata de un modelo totalmente insostenible, al borde de la quiebra y con consecuencias que ya son evidentes.

\section{Consecuencias de un sistema lineal}

Investigaciones previas realizadas por la Ellen MacArthur Foundation (2015) y Andrews (2015) arrojan una serie de consecuencias que ya tienen repercusiones, entre las más destacadas se encuentran:

\section{Pérdidas económicas y residuos estructurales}

Como se describió en el apartado anterior, anualmente se están generando cifras impactantes de residuos sólidos, de los cuales un 33\%, aproximadamente, no se tratan de ninguna manera (Banco Mundial, 2016). Sin embargo, se ha evidenciado una generación de residuos estructurales en sectores optimizados. Un ejemplo 
de esto es la industria automotriz, en donde se estima que un coche pasa alrededor del $90 \%$ del tiempo aparcado, ya sea en trancones o en casa; o en la industria alimenticia, en donde un $31 \%$ de los alimentos se está perdiendo a lo largo de la cadena de valor. Todos estos casos no solo tienen implicaciones económicas notables, sino que también se identifican fallas en sus cadenas de suministro que están permitiendo tales desperdicios.

\section{Riesgos de precios}

Se ha evidenciado cómo un sistema basado en la extracción, producción y consumo masivo aumenta la exposición a riesgos en las empresas, más específicamente variaciones en la volatilidad de los precios de los recursos naturales, y los problemas en la cadena de suministro al presentarse escasez o deterioro de las materias. Estas variaciones pueden generar, a su vez, una sobrecarga en el crecimiento económico, al aumentar la incertidumbre, disminuir la inversión y subir los costos de protección.

\section{Riesgos de suministro}

Las regiones que poseen recursos naturales no renovables en el mundo no son demasiadas, por lo que la mayoría de las naciones tiende a depender de las importaciones de materias primas. Adicional a esto, se observa un aumento del riesgo para la seguridad del suministro. Todo esto es una consecuencia directa de la escasez y de fallas en la optimización del uso de estos recursos.

\section{Deterioro de los sistemas naturales}

Teniendo en cuenta que actualmente la riqueza global a largo plazo depende de la explotación de recursos bajo este sistema lineal, se observa una afectación notoria en la productividad global proveniente del agotamiento de estas reservas naturales y del capital natural, cuyas causas de agotamiento se ven reforzadas por el cambio climático, la degradación de suelos, la pérdida de biodiversidad y la contaminación, entre otros factores.

\section{Aumento de la población e incremento en el PIB}

Un crecimiento económico acelerado trae consigo un aumento en la riqueza de los países, lo que se refleja en un incremento del producto interno bruto. Aunque esto conlleva al desarrollo científico y tecnológico, que a su vez reduce la mortalidad infantil e incrementa la expectativa de vida, implica igualmente un crecimiento demográfico acelerado que se soporta en el consumo y la explotación masiva de recursos. De acuerdo con las Naciones Unidas (2019), la población mundial pasó 
de ser un billón, en 1804, a 7.7 billones, en 2019, con una proyección de 9.7 billones para el 2050. Estas cifras no solo constatan el aumento en la demanda de recursos, la escasez y la inevitable desaparición de algunos de estos bienes naturales, sino que también dan razón a la necesidad de cambiar modelos e innovar para un desarrollo sostenible de todos y para todos.

\section{Economía circular}

La naturaleza sostiene la vida universal de todos los seres. TENZIN GYATSO (decimocuarto dalái lama)

Como se pudo evidenciar en la sección anterior, aunque el crecimiento económico impulsado por el sistema lineal nos ha traído avances y oportunidades únicas, no es un sistema sostenible. Desde la década de los setenta la humanidad ha sido testigo de la necesidad de cambio, con sucesos como la crisis de petróleo y el primer registro de un sobregiro de la Tierra en esa misma década, surgió la necesidad de empezar a indagar en posibles alternativas a este sistema lineal.

Entre los primeros registros que se tiene sobre la noción de economía circular se encuentra el de Boulding (1966), para quien resultaba ideal la implementación de un sistema ecológico cíclico que frenara los efectos del sistema lineal. Más adelante, Meadows y otros (1972), en Limits to Growth, demostraron por qué un sistema basado en la extracción, producción, uso y desecho continuo de recursos no era sostenible y cómo su continuidad implicaba alcanzar los límites planetarios en los próximos cien años. Otro de los aportes más notorios se dio en 1982, cuando Walter Stahel introdujo el término closed loop, o cierre de ciclos, y dio inicio a la interpretación de un sistema de 6 eres (reutilizar, reciclar, rediseñar, remanufacturar, reducir, recuperar), base del sistema circular.

Sin embargo, uno de los hitos que transformó la investigación en sostenibilidad y circularidad se dio gracias al Informe de Brundtland (Keeble, 1987), presentado por Gro Harlem Brundtland, ex primera ministra de Noruega, ante la primera Comisión Mundial para el Medio Ambiente y el Desarrollo de la ONU, en 1987. En este informe se formuló un análisis y un replanteamiento a las políticas de desarrollo económico y surgió el término Desarrollo Sostenible, planteado como "aquel que satisface las necesidades del presente sin comprometer la capacidad de las generaciones del futuro". Desde ese entonces se ha venido formulando una estructura más concisa en torno a la implementación de este sistema circular, pasando de ser un concepto especializado a un movimiento mainstream o convencional aplicable. 
De acuerdo con lo anterior, de manera muy completa, para Suárez-Eiroa y otros (2019) la economía circular es

\begin{abstract}
un sistema de consumo de producción regenerativa que tiene por objeto mantener las tasas de extracción de recursos y las tasas de generación de desechos y emisiones bajo valores adecuados a las fronteras planetarias, mediante el cierre del sistema, la reducción de su tamaño y el mantenimiento del valor del recurso el mayor tiempo posible dentro del sistema, apoyándose principalmente en el diseño y la educación, y con capacidad para ser implementado a cualquier escala. (p. 14)
\end{abstract}

Teniendo en mente esta definición y los planteamientos previamente expuestos, a continuación, se explican en detalle los principios y características de este sistema; asimismo, se hace una descripción del diagrama de mariposa, el esquema que describe a la economía circular, así como las ventajas y desventajas de su implementación y un caso de éxito de su respectiva aplicación.

\title{
Principios y características
}

Con base en estudios realizados por la Ellen MacArthur Foundation (2015), se han identificado tres principios que rigen la economía circular, estos son:

Principio 1: preservar y mejorar el capital natural controlando reservas finitas y equilibrando los flujos de recursos renovables. Para lograr un control de reservas, en una situación donde se necesiten recursos, lo ideal es que un sistema circular seleccione tecnologías y procesos que trabajen con recursos renovables (Geisendorf \& Pietrulla, 2017). Adicionalmente, esta economía promueve un movimiento constante de flujos de nutrientes dentro del sistema, lo que, a su vez, motiva la regeneración de los sistemas bióticos, hecho que se refleja en una mejora del capital natural.

Principio 2: optimizar los rendimientos de los recursos distribuyendo productos, componentes y materias con su utilidad máxima en todo momento tanto en ciclos técnicos como biológicos. Esto se traduce en reestructurar las técnicas de diseño y fabricación para garantizar que los materiales y demás componentes puedan recircular a través del reciclaje, remanufactura o recuperación. Igualmente, un sistema circular garantiza que los ciclos de vida de los materiales se maximicen, ya sea desde el ciclo biológico o el ciclo técnico, de manera que, aunque su utilidad aumente, su eficiencia y eficacia no disminuyan con el tiempo, y que cuando su ciclo de vida termine, se conviertan en materia prima para otras industrias (Bonciu, 2014).

Principio 3: promover la eficacia de los sistemas detectando y eliminando del diseño los factores externos negativos. Esto implica modificaciones en los sistemas, de manera que se reducen los daños y riesgos en factores como movilidad, centros 
de acogida y sanidad. Igualmente, se promueve una gestión sostenible de factores externos como el uso del suelo y la contaminación de fuentes. Bajo este principio, entra a jugar un papel clave para el éxito la interacción entre industrias y usuarios que se comprometan a implementar la circularidad, e igualmente se pasa de un sistema de decisión individual a uno de decisión colectiva, por lo que son necesarios cambios en la educación, valores y comportamientos de los productores y consumidores (Bonciu, 2014).

Tomando como base los tres principios descritos anteriormente, se logra articular un sistema circular. Sin embargo, los fundamentos bajo los que debería funcionar son:

1. Reintroducción de los residuos en los ciclos de producción, en donde materias biológicas no tóxicas son devueltas al sistema natural mediante compostaje, digestión anaerobia o procesos similares, y materiales técnicos son recuperados, remanufacturados o reciclados maximizando su valor en el tiempo (Ellen MacArthur Foundation, 2015). Igualmente, en una economía circular se implementa el modelo de las 6 eres, en donde se transforman los procesos de producción para convertirse en ciclos sostenibles. De acuerdo con Jawahir \& Bradley (2016), la metodología se basa en:

- Reducir: donde se reduce el uso tanto de recursos en la preproducción, como son energía, materias primas y otros, como las emisiones y los posibles desechos.

- Reusar: se refiere a la reutilización de un producto, o sus partes, después de su primer ciclo de vida.

- Reciclar: se trata de transformar en nuevos materiales o productos, materiales que hubieran sido considerados en un primer momento como basura.

- Recuperar: en donde se recolectan productos que están finalizando su etapa de uso, se desensamblan, clasifican y limpian para entrar a un nuevo ciclo de vida póstumo.

- Rediseñar: etapa en donde se rediseñan las siguientes generaciones de productos hechos a base de materias y recursos recuperados de otros ciclos, teniendo en cuenta su maximización de vida útil y eficiencia.

- Remanufacturar: donde productos usados se reprocesan para su restauración a un estado original como un nuevo producto, tratando de reutilizar tantas partes como sea posible. 
2. Resiliencia a través de la diversidad, en donde, al igual que como funcionan los ecosistemas, la diversidad actúa como impulsora de la restauración y resiliencia ante el cambio, y se alimenta de conexiones, recursos, relaciones con usuarios e innovaciones (Joustra et al., 2013). Un ejemplo de esta resiliencia en un sistema circular ante épocas de crisis se refleja en los aportes de las empresas en cuanto a eficiencia e innovación. Según la Ellen MacArthur Foundation (2015), "las empresas más grandes aportan volumen y eficiencia, mientras que las pequeñas ofrecen modelos alternativos cuando hay crisis" (p. 8).

3. Energía proveniente de fuentes renovables, ya que todo sistema requiere de energía, una economía circular se formula alrededor de aprovechar fuentes como el Sol, el viento, los mares, entre otras fuentes, para el funcionamiento de estos ciclos, más específicamente en las etapas de recuperación y remanufacturación, en donde se depende de energía física y de labor para su procesamiento (Joustra et al., 2013).

4. Pensar de manera holística y en sistemas. Esto proviene del hecho de que muchos elementos del mundo hacen parte e interactúan entre sistemas, desde las plantas y animales hasta las personas (Ellen MacArthur Foundation, 2015). Para lograr una transformación efectiva del sistema lineal actual al circular, es necesario comprender todos estos tipos de interacciones para crear ciclos eficientes.

\section{El diagrama de mariposa}

Como se ha planteado anteriormente, una economía circular se basa en una transición de flujos lineales a ciclos en donde las pérdidas de una etapa de producción se vuelven insumos de la siguiente. Para un mejor entendimiento, investigadores de la Ellen MacArthur Foundation plantearon un diagrama del sistema o diagrama de mariposa, en donde se plasman los diferentes flujos continuos de materiales, tanto técnicos como biológicos, a través de cinco ciclos de valor: económico, de fabricación, social, ambiental o humano.

En cuanto a los tipos de flujos, en la figura 2 se pueden observar los flujos biológicos en el ala derecha de la mariposa, estos representan las reservas de materias renovables y es donde se produce el consumo y se regeneran los nutrientes. Por otro lado, los flujos técnicos se pueden observan en el ala izquierda de la mariposa, estos flujos representan las reservas finitas y es donde se presenta el uso de las materias. 


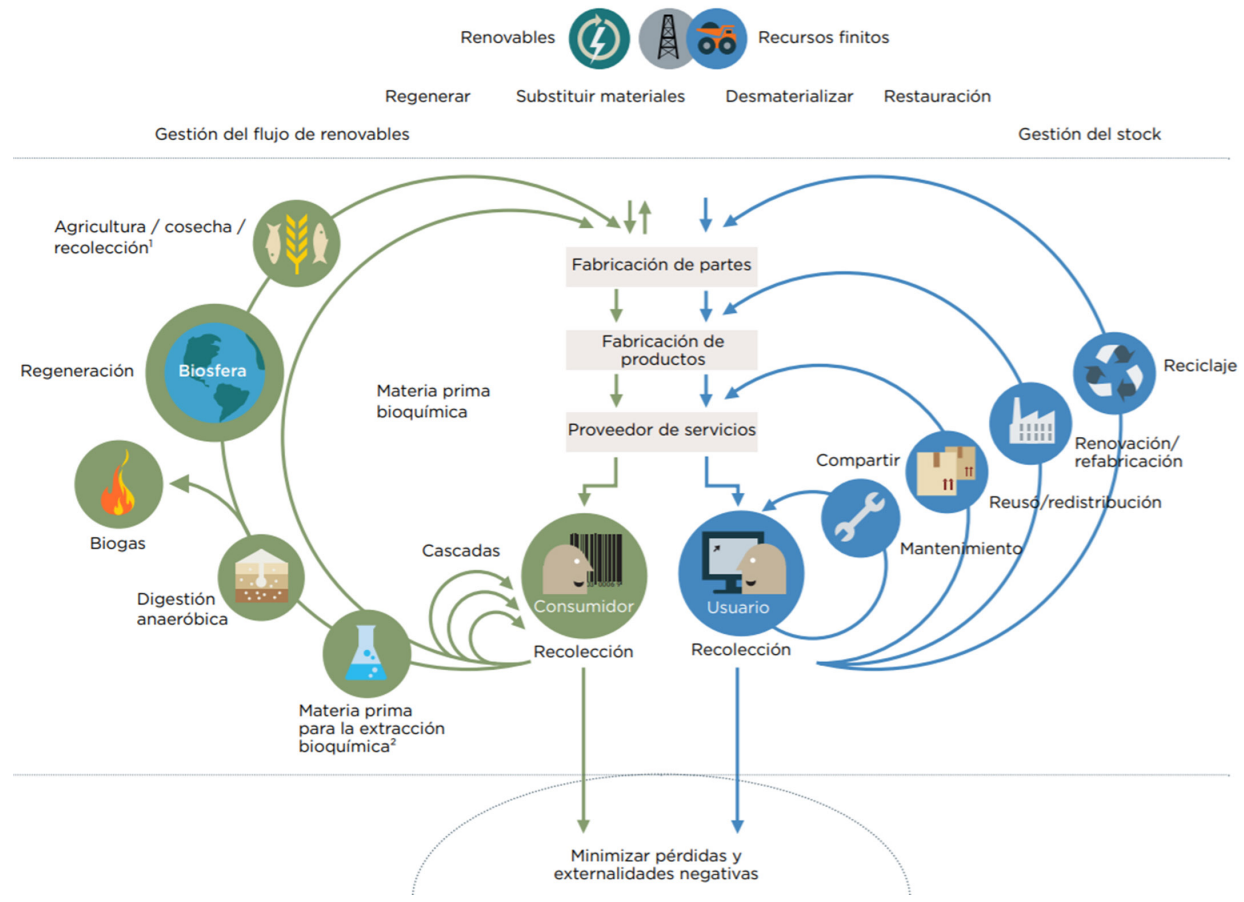

Figura 2. Diagrama de mariposa de la economía circular Fuente: Ellen MacArthur Foundation (2015)

Como se puede observar en el diagrama, los únicos insumos que se tienen son las fuentes renovables y los recursos finitos; en cuanto a los flujos técnicos, se encuentran las principales acciones encaminadas a cerrar el ciclo de los residuos (previamente descritas), que suelen ir al final bajo un sistema lineal, como son el reciclaje, la renovación, la remanufacturación, el reuso, y el mantenimiento de productos. Por otro lado, en los flujos biológicos se aprecia la materia prima bioquímica, la cual se puede retornar a la biósfera, como en el caso de los productos de agricultura, cosecha y recolección, los materiales que retornan al suelo para su restauración, los procesos de digestión anaerobia y la materia prima necesaria para la extracción bioquímica. Finalmente, como se observa en la parte inferior de la ilustración, existen pérdidas y externalidades negativas provenientes de cada flujo, que, aunque no pueden ser totalmente evitadas, deben minimizarse. 


\title{
Economía Circular en Colombia
}

\author{
Hemos aprendido que, el crecimiento económico \\ y la protección del medio ambiente pueden y deben ir de la mano. \\ CHRISTOPHER DODD (político estadounidense)
}

Un país como Colombia, con su prolífica biodiversidad, múltiples culturas y espíritu emprendedor característico de sus habitantes, no debe ignorar la oportunidad de implementar un sistema circular que proteja sus valiosos recursos y garantice una estabilidad ambiental, económica y social a largo plazo. Es por esto que resulta esencial una constante organización de los poderes público y privado que, a través de políticas, inversiones, consultorías e investigación, y tomando en cuenta las necesidades y el contexto colombiano, integren un modelo cooperativo para el desarrollo de la economía circular en el país.

\section{Políticas para la economía circular}

Colombia ha venido trabajando en políticas para la regulación de los efectos del cambio climático, problemáticas ambientales y desarrollo sostenible desde finales de los años noventa; sin embargo, en cuanto a políticas o propuestas encaminadas específicamente a la economía circular, fue solo hasta el año 2019 que se presentó la Estrategia Nacional de Economía Circular, primer estudio de este tipo en Latinoamérica (Gobierno de la República de Colombia, 2019). No obstante, el país cuenta con diferentes políticas y lineamientos que han funcionado como marco regulatorio para la economía circular; Rozo (2019) recopiló estas políticas, de las cuales las más trascendentales son:

- Política para la gestión integral de residuos, presentada por el Ministerio de Ambiente en 1997.

- Decreto 389. Política de parques industriales ecoeficientes (PIE), enfocada en la creación de parques industriales que desarrollan proyectos dirigidos a mejorar su desempeño económico, ambiental y social, presentada por la Alcaldía Mayor de Bogotá en el año 2003.

- Lineamientos y estrategias para fortalecer el servicio público en el marco de la Gestión Integral de Residuos Sólidos, presentada por el Departamento Nacional de Planeación en el 2008.

- Política Nacional de Producción y Consumo Sostenible, enfocada en una competitividad empresarial y un mejoramiento ambiental bajo 
mercados verdes, presentada por el Ministerio de Ambiente, Vivienda y Desarrollo Territorial en 2010.

- Política Nacional para la Gestión Integral de Residuos Sólidos-CONPES del 2016 presentada por el Ministerio de Ambiente en 2016.

- Proyecto de ley 175 del 22 de agosto de 2018, en donde se establece, a partir del año 2030, la prohibición de plásticos de un solo uso y disposiciones semejantes que permitan su sustitución y cierre de ciclos, presentada ante la Cámara de Representantes y archivada de momento.

- Política de 'Crecimiento Verde', presentada por el Departamento de Planeación Nacional (2018) cuyo objetivo es "impulsar a 2030 el aumento de la productividad y la competitividad económica del país, al tiempo que se asegura el uso sostenible del capital natural y la inclusión social, de manera compatible con el clima” (párr. 1).

\section{Estrategia nacional de economía circular}

Producto de una convergencia de múltiples actores del sector privado, público, académico y de la sociedad civil de todas las regiones de Colombia, esta estrategia plantea mecanismos de gestión y política pública que facilitarán a las entidades del Estado la transición hacia este nuevo sistema. De acuerdo con lo propuesto en el documento de la Estrategia Nacional de Economía Circular, desarrollada por el Ministerio de Ambiente y Desarrollo Sostenible, el Ministerio de Comercio Industria y Turismo, y el Gobierno Nacional de Colombia (2019), las principales formulaciones son:

- Innovación en mecanismos normativos que fomentan un cambio del sistema tradicional de producción en empresas y emprendimientos.

- Gestión de incentivos que promueven procesos de transformación de sistemas industriales y agrícolas a través de apoyos en capacitación y asistencia técnica

- Promoción de investigación, innovación y generación de conocimiento en dichos temas.

- Desarrollo de un sistema de información sobre economía circular para el seguimiento a la implementación de la estrategia y la medición del avance del país en el tema.

- Cooperación internacional para intercambio de tecnologías y conocimiento. 
Igualmente, a través de esta estrategia se plantea un enfoque en seis líneas de acción donde se fijan metas a plazos e indicadores de cumplimiento y avance. Estas líneas son flujos de materiales industriales y productos de consumo masivo, flujos de materiales de envases y empaques, flujos de biomasa, fuentes y flujos de energía, flujos de agua y flujos de materiales de construcción (Ministerio de Ambiente y Desarrollo Sostenible, Ministerio de Comercio Industria y Turismo, y Gobierno Nacional de Colombia, 2019).

\section{Casos exitosos}

Motivadas por las tendencias mundiales orientadas hacia la aplicación de los fundamentos de la economía circular, especialmente en países como Holanda, Dinamarca Alemania, Japón, entre otros, muchas empresas colombianas han decidido emprender una transformación en sus sistemas productivos e iniciar una transición hacia un sistema circular. Una de las iniciativas más reconocidas actualmente es RedES-CAR (Red de Empresas Sostenibles-Corporación Autónoma Regional de Cundinamarca), la cual se constituye como una alianza entre los sectores público, privado y la academia para promover la transformación productiva de las empresas mediante la aplicación de estrategias de cambio a través de diferentes rutas: producción más limpia, simbiosis industrial y gestión integral del agua (RedES-CAR, s. f., a).

Como base de su metodología, RedES-CAR se fundamenta en tres pilares: estrategia de cambio, capacidades y empoderamiento, y colaboración en red, tal como se puede observar en la figura 3. Con base en estos tres pilares se estructura su metodología, que consiste en una conformación de cadenas, en donde se identifican posibles actores y se establecen convocatorias; desarrollo de talleres para el fortalecimiento de capacidades, en donde se intercambian experiencias y se diseñan proyectos de producción más limpia (PML); simbiosis industrial o gestión integral del agua, en donde se genera un seguimiento a las empresas a través de encuestas y talleres; $y$, finalmente, si el proceso ha sido exitoso, se le realiza un reconocimiento a la empresa exaltando su papel en la transformación productiva.

Esta alianza ya cuenta con más de 300 proyectos encaminados a una transformación a favor de la sostenibilidad, en sectores económicos como el agropecuario, alimentos y bebidas, comercio y construcción, entre otros.

Un ejemplo representativo de los proyectos que se encuentra gestionando RedES-CAR es el cambio de fuente de abastecimiento de agua para procesos secundarios de Productos Ramo S.A., un proyecto enfocado en PML, en donde el 


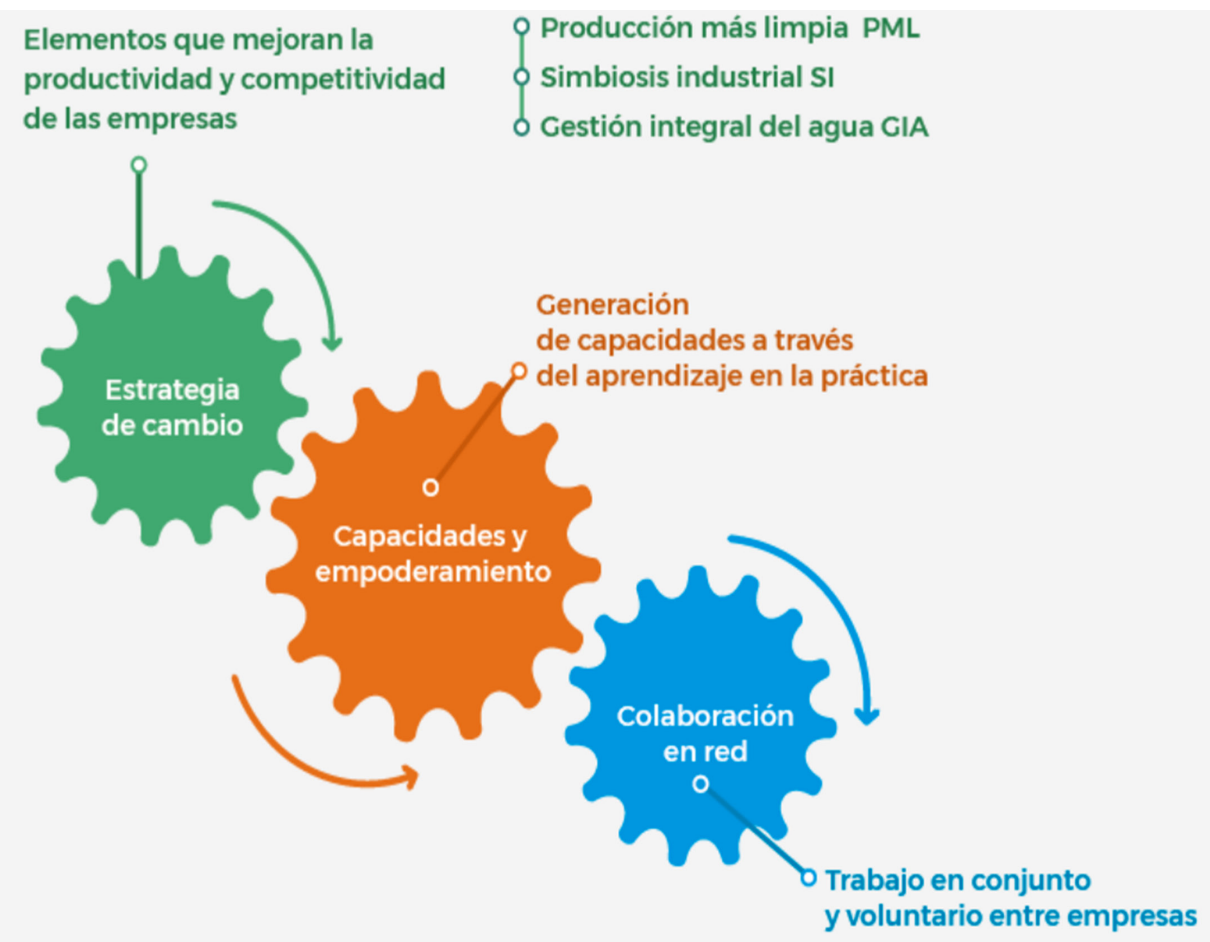

Figura 3. Pilares de la metodología de RedES-CAR

Fuente: RedES-CAR (s. f., b)

consumo de agua anual es de $25.550 \mathrm{~m}^{3} /$ año. De acuerdo con Cuervo \& Padilla (s. f.) su objetivo es "mejorar el sistema de captación de aguas lluvias y conducirlas a un tanque principal de almacenamiento, buscando reducir en $8267 \mathrm{~m}^{3} /$ año el volumen de captación de agua subterránea y reutilizar $4680 \mathrm{~m}^{3} /$ ańo de agua de lavado de filtros de la planta de tratamiento de procesos (PTAP)" (p. 1).

\section{Gestión ambiental en el Ejército Nacional de Colombia}

Dentro de algunas décadas, la relación entre el ambiente, los recursos y los conflictos será tan obvia como la conexión que vemos ahora entre derechos humanos, democracia y paz. wangari maAthai (Premio Nobel de Paz 2004)

El Ejército Nacional de Colombia, siguiendo sus principios de compromiso y fe en la causa, vela por la seguridad de la nación, así como por la protección y bien- 
estar de sus recursos naturales y medioambiente (Ejército Nacional de Colombia, s. f.). Bajo el ámbito ambiental, tiene un compromiso con la Convención Marco de las Naciones Unidas para el Cambio Climático, el "Pacto por la Sostenibilidad: Producir Conservando y Conservar Produciendo", parte del Plan Nacional de Desarrollo 2018-2022; y los lineamientos del Ministerio de Defensa Nacional, con la política de Defensa de Seguridad del 2019 y su objetivo 614: preservar y defender el agua, la biodiversidad y los recursos naturales como activos estratégicos de la nación (Tovar, 2020).

Adicionalmente, en el Plan Estratégico Militar 2030 se garantiza un compromiso con el desarrollo sostenible mediante empleo de capacidades militares del Objetivo Estratégico No. 5 y la protección y preservación del medioambiente en Colombia establecido en el Objetivo Específico No. 3, del Objetivo Estratégico No. 6 (Comando General Fuerzas Militares de Colombia, 2015).

\section{Acciones a favor de la sostenibilidad}

A partir de los lineamientos a los que el Ejército Nacional decidió adherirse, con el objetivo de estructurar un sistema de gestión ambiental, surgieron diferentes áreas de enfoque bajo las cuales se constituyeron operaciones y planes de acción. A este respecto, el TC. Tovar (2020) explica estas áreas y sus principales acciones en su conferencia sobre la gestión ambiental en el Ejército Nacional, estas son:

- Ecosistemas: en donde se diseñan y ejecutan proyectos encaminados a la preservación de ecosistemas. Entre los más destacados están:

- Proyecto Restauración Natural Asistida en Cerro Medellín, donde se tiene la meta de sembrar más de 20.000 unidades forestales.

- Aeroforestación, para reforestar y restaurar zonas degradadas en parques naturales cercanas a San José del Guaviare y El Retorno (Guaviare).

- Plan de Recuperación Ambiental, orientado a la protección de ecosistemas estratégicos. Sus 6 metas son:

1. Siembra de más de cinco millones de árboles para 2022.

2. Propagación y siembra de un millón de frailejones y 39 invernaderos.

3. Propagación y siembra de medio millón palmas de cera y 7 viveros.

4. Reforestación de zonas afectadas por la minería ilegal.

- Creación de dos macroviveros para la producción de tres millones de plántulas para la recuperación del ecosistema del Parque de Chibiriquete y Tinigua.

- Protección y conservación de 373 fuentes hídricas. 
- Educación ambiental: en donde se ha capacitado a más de 7000 soldados, 64 suboficiales tecnólogos y 281 gestores ambientales en cada una de las unidades militares para las labores de atención y preservación del medioambiente.

- Saneamiento básico: planes de diseño y mantenimiento de pozos, redes hidráulicas y sanitarias de los diferentes planteles del Ejército Nacional de Colombia.

- Trámites legales: periódicamente se realizan auditorías ambientales a las unidades militares para darle seguimiento a todos los procesos, los impactos ambientales y sus respectivos controles.

Adicional a estas acciones, en 2019 se empezó a ejecutar la Operación Mayor Artemisa, catalogada como la misión más ambiciosa en términos ambientales realizada por el Ejército Nacional de Colombia, y cuya finalidad es la defensa de la biodiversidad, de la selva tropical y de los parques nacionales (Presidencia de la República, 2019). De esta manera, las líneas de acción de la operación son (Tovar, 2020):

- Control de tráfico ilegal de fauna y flora.

- Control de tráfico ilegal de madera.

- Actividades de acción integral con autoridades ambientales.

- Reforestación.

- Control de incendios forestales.

- Control de cultivos ilícitos.

- Control de minería ilegal.

- Plan de Recuperación Ambiental 2020-2022.

Finalmente, para afianzar el compromiso con la gestión ambiental y el desarrollo sostenible de Colombia, el Ejército ha venido implementando en sus escuelas de formación, especializaciones y maestrías orientadas en estos temas. Igualmente, ha fomentado la investigación y participación de la comunidad académica en proyectos de índole ambiental, ejemplo de esto fue el 'Primer Encuentro por el Desarrollo y Sostenibilidad Ambiental' organizado por la Escuela de Armas Combinadas y la Escuela de Aviación, en donde investigadores y expertos en desarrollo y medioambiente formularon iniciativas para fortalecer la gestión ambiental en el Ejército Nacional. 


\title{
Oportunidades de Economía Circular en el Ejército Nacional de Colombia
}

\author{
El liderazgo sustentable refleja la conciencia emergente entre \\ las personas que están decidiendo vivir sus vidas y dirigir sus \\ organizaciones en una forma que tome en cuenta su impacto \\ en el planeta, la sociedad y la salud de economías locales y globales. \\ MARY A. FERDIG (consultora en liderazgo y desarrollo internacional)
}

Partiendo de las premisas bajo las que se constituye una economía circular, pueden surgir múltiples iniciativas para la transformación de los procesos que suceden en empresas e instituciones, hacia un modelo sostenible y viable. En lo que respecta a las FF. MM., múltiples iniciativas se han desarrollado mundialmente, desde planes de acción enfocados en la participación de las FF. MM. en temas de seguridad ambiental en Estados Unidos (Glenn et al., 1998), hasta el uso de estrategias de creación de valor compartido en organizaciones públicas como el Ejército, para mejorar su competitividad básica en Corea del Sur (Kwon \& Park, 2019). Igualmente, estudios encaminados a proponer mejoras para diferentes sistemas de gestión en las FF. MM. toman cada vez mayor impulso, como es el caso de la 'Propuesta para el mejoramiento de la Cadena de Suministros y Abastecimiento del Ejército Nacional de Colombia, para responder a las necesidades del siglo XXI' (Restrepo, 2014).

Tomando los diferentes contextos y objetivos de las iniciativas previamente descritas, a continuación, se presenta un análisis metodológico y teórico para la identificación de posibles oportunidades de economía circular en las instalaciones del Ejército Nacional de Colombia.

\section{Análisis de casos}

Con base a lo dicho anteriormente, se realizó una revisión bibliográfica enfocada en planes y estrategias que diferentes FF. MM. están aplicando, así como estudios sobre su implementación. Igualmente, se realizó una revisión enfocada en los poderes ambientales o de ejecución que se les da a la FF. MM. Con base en los resultados, se decidió dividir el presente análisis en tres secciones: rol ambiental, planes de sostenibilidad y ejemplos de aplicación de alternativas. A continuación, se presenta un resumen para cada sección como lo muestran las tablas 1, 2 y 3. 


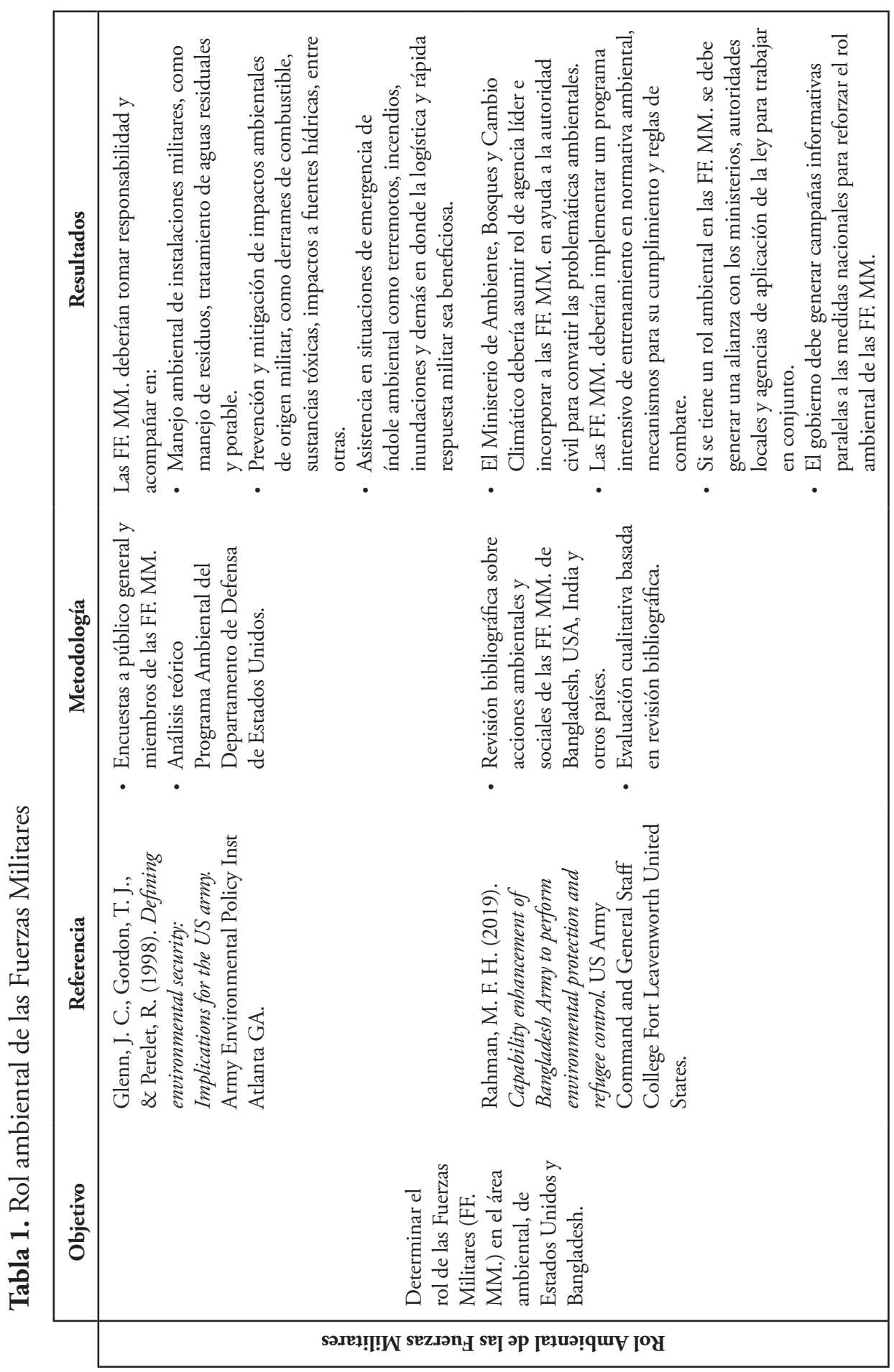




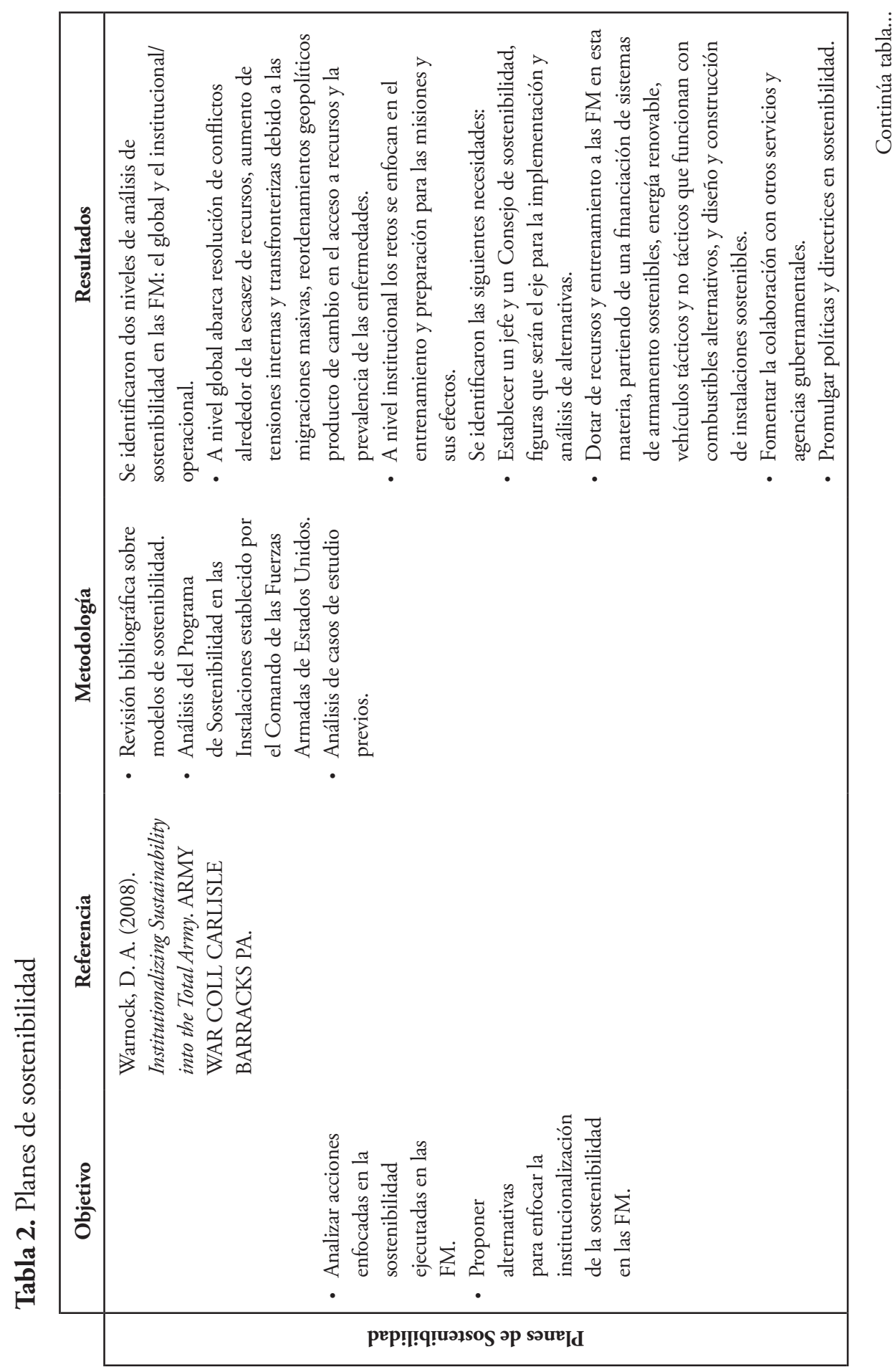




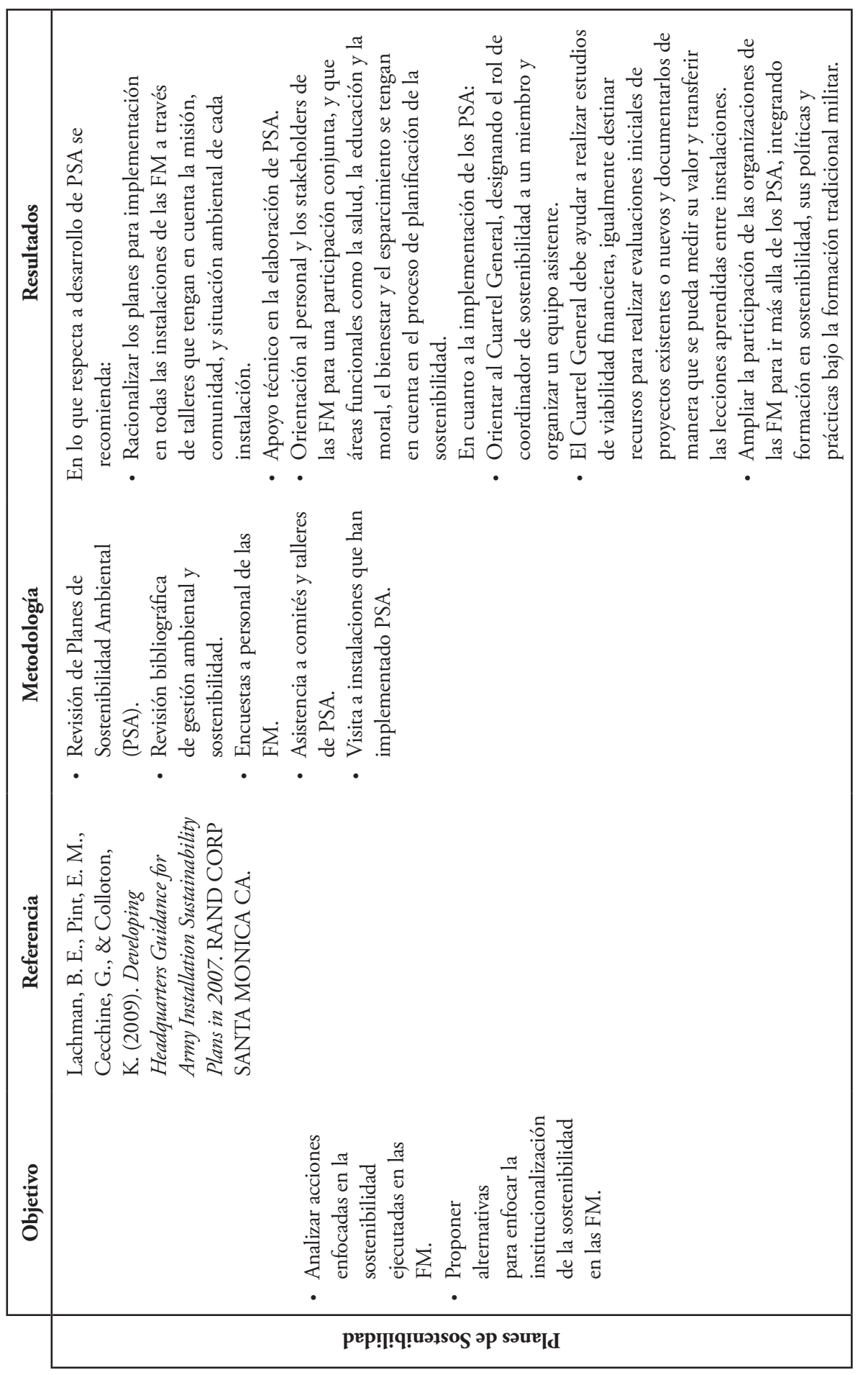




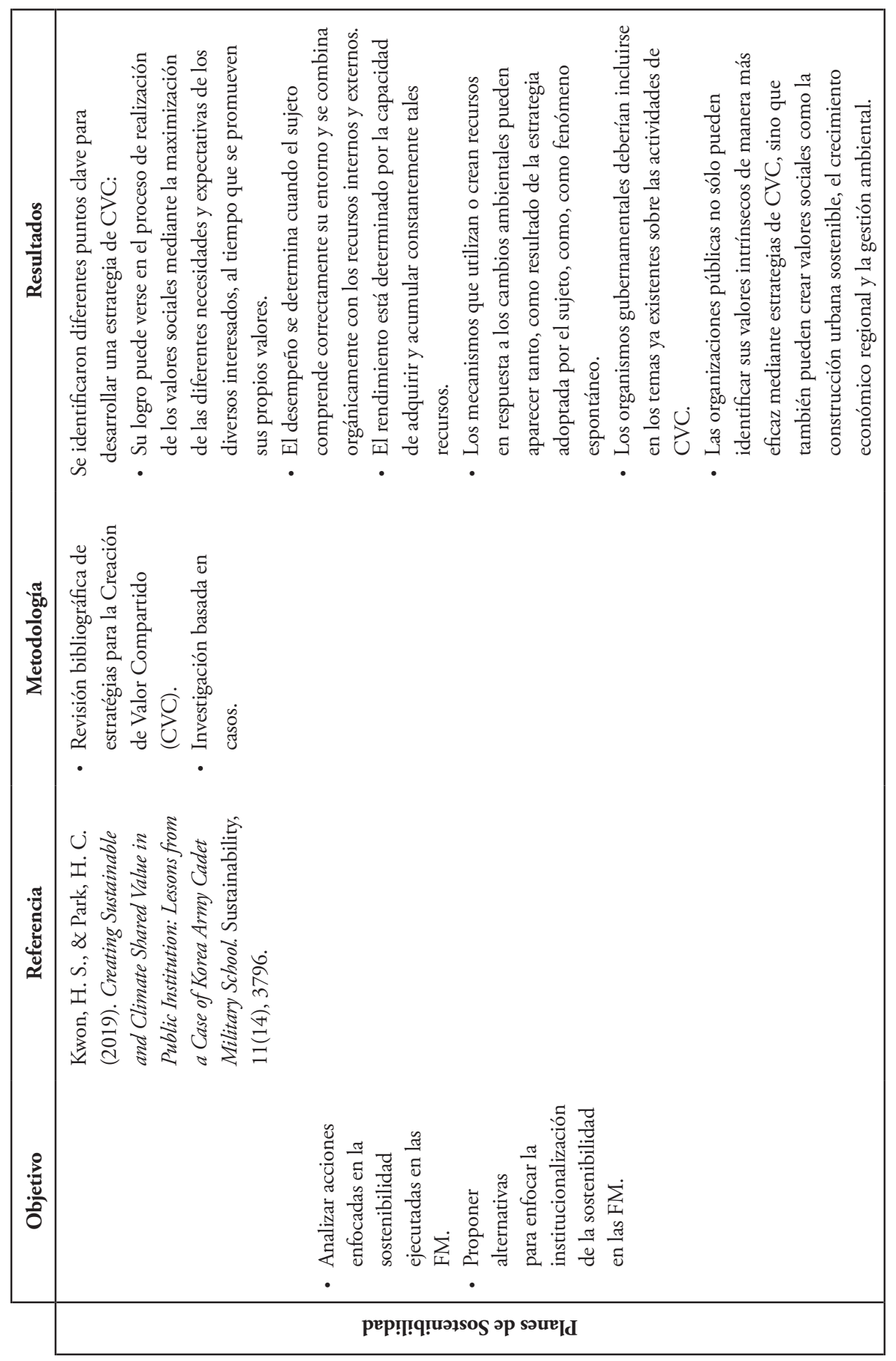




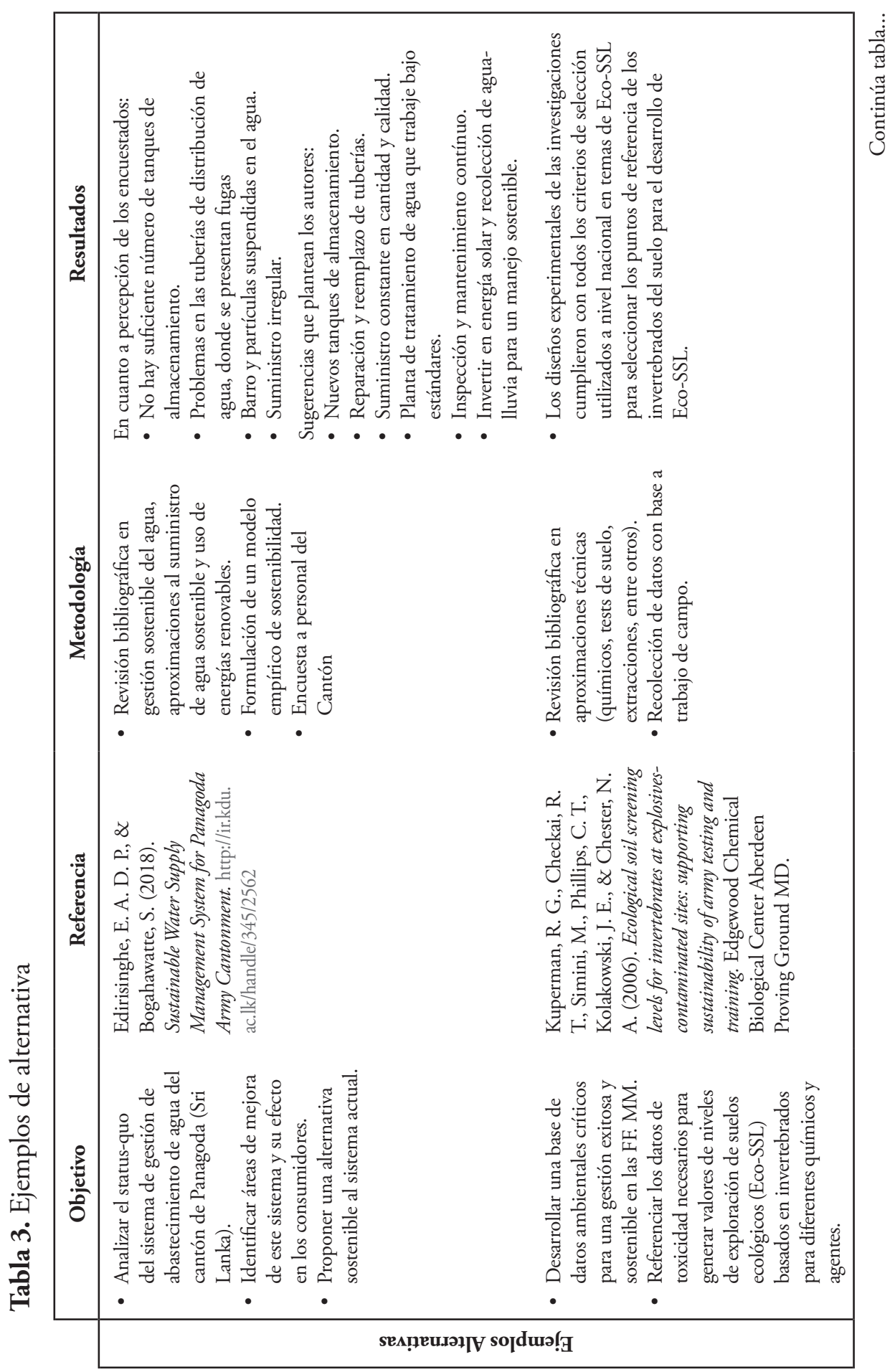




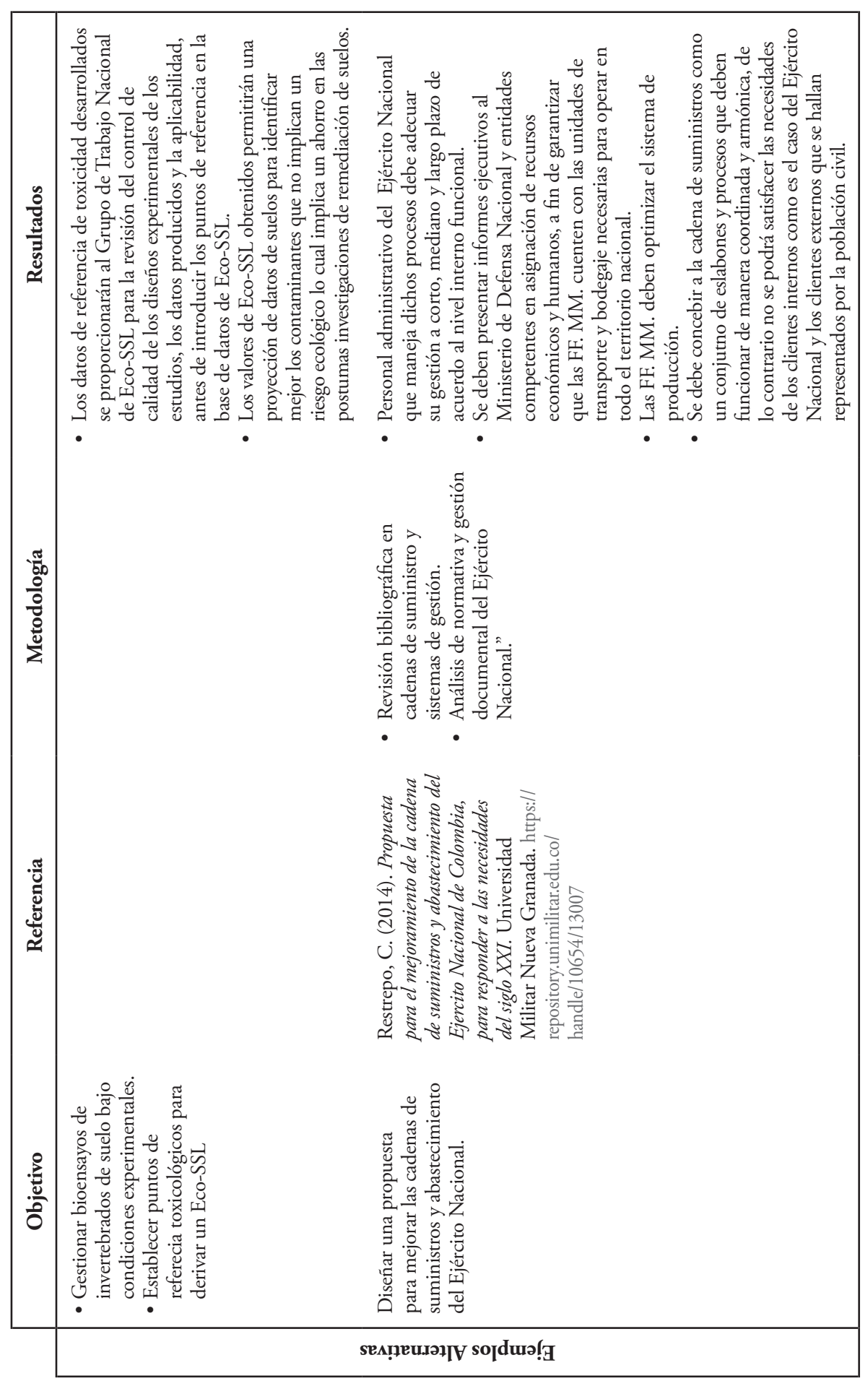


Como se puede observar, entre los principales roles que las FF. MM. deben adoptar en el ámbito ambiental se encuentran el de propiciar la preservación y mitigación de todos los posibles impactos ambientales que puedan surgir de sus actividades, dar asistencia ante desastres naturales y demás problemáticas colectivas, y generar alianzas con ministerios y otras entidades gubernamentales para forjar un trabajo conjunto de protección y gestión ambiental.

En lo que respecta al análisis de los planes de sostenibilidad que han ejecutado diferentes instituciones militares del mundo, se resaltan como medidas para una correcta implementación: tener en cuenta los conflictos más allá de los ambientales, como lo son los problemas de migraciones, desempleo, educación, bienestar y salud de la población; incluir en el entrenamiento y formación militar una orientación hacia la gestión de proyectos encaminados al desarrollo sostenible; y generar cargos oficiales y consejos de sostenibilidad para una gestión de proyectos, entre otros.

Finalmente, se presenta un conjunto de ejemplos sobre iniciativas ambientales y de mejora a los sistemas de suministro y abastecimiento del Ejército. Parte de la idea de presentar estos ejemplos es analizar su metodología, posible aplicación de principios circulares y viabilidad. De esta manera, en lo que respecta a la investigación del sistema de gestión de abastecimiento en un Cantón de Bangladesh, realizada por Edirisinghe \& Bogahawatte (2018), se presenta la intención de modificar el sistema actual por uno que funcione de manera sostenible, con energías renovables y mejor eficiencia. Para su estudio se tuvo en cuenta la opinión del personal del cantón, a partir de allí se formula un modelo empírico para medir la sostenibilidad del proyecto y se referencian otros casos exitosos. Todo esto refleja la intención de cambiar el modelo a uno sostenible, sin embargo, los investigadores se quedan cortos a la hora de demostrar la viabilidad del proceso. Caso similar sucede en la investigación de Restrepo (2014), quien a través de un análisis teórico plantea una mejora a la cadena de suministro y abastecimiento del Ejército, sin embargo, aunque describe mejoras logísticas que debe implementar la institución, falla al enunciar los aspectos ambientales y de sostenibilidad que deberían tenerse en cuenta para una cadena de suministro y abastecimiento eficientes, lo que vuelve inviable la inclusión de su propuesta en un modelo circular.

\section{Implementación de estrategias}

Teniendo como referencia el análisis previo, desde las recomendaciones para la implementación de iniciativas sostenibles en las instalaciones de las FF. MM., hasta los ejemplos de las diferentes investigaciones en torno a soluciones soste- 
nibles, se puede iniciar una estructuración de un modelo circular, en donde se analicen los diferentes sistemas en que se componen las instalaciones del Ejército, sus cadenas de suministro, residuos y energía. De esta manera, Van Hoof y otros (2018) recomiendan "actuar sobre factores que puede afectar el desempeño del aparato productivo, dentro de los cuales se consideran: tecnología e innovación, desarrollo de capacidades y participación en cadenas productivas" (p. 63).

En lo que respecta a tecnología e innovación, se prosigue a analizar con qué tipo de tecnologías cuentan las instalaciones, cuales se están empleando en modelos de innovación sostenibles y cómo están aportando a la productividad de la institución, entendiendo que esta última se optimiza involucrando tecnologías e innovación en los procesos, y que las instituciones pueden generar valor agregado implementándolas (Van Hoof et al., 2018).

Como se mencionó en el análisis, el desarrollo de capacidades en el personal del Ejército Nacional de Colombia es un punto fundamental para que todo modelo sea exitoso, por esto, Van Hoof y otros (2018) describen como "solo mediante la adecuada capacitación del recurso humano, una empresa puede adquirir el conocimiento, la especialización y el compromiso para asegurar la eficiencia de los procesos e identificar oportunidades de mejoramiento" (p. 65).

En cuanto a la participación en cadenas de valor, los autores destacan cómo, a través de la incursión de la institución en una cadena de suministro activa y organizada, se promueve también la generación de confianza y desarrollo de procesos de colaboración, lo que influye en la capacidad productiva de los demás actores que hacen parte de la cadena (Van Hoof et al., 2018).

Finalmente, a la hora de implementar metodologías para gestionar la transformación hacia un modelo circular, se debe entender cómo cada actor del sistema funciona y se relaciona con los demás. Así, no se puede implementar un proyecto sin que se tenga en cuenta: la situación bajo la cual el personal del Ejército intervendría; la existencia de otras organizaciones que podrían participar para lograr una mejor eficiencia y cohesión; los impactos económicos que le traería a la institución; y los demás efectos que vienen de la mano con estos modelos. Para esto, se recomienda seguir metodologías como la RedES-CAR, descrita previamente, y que se apoya en un entendimiento de múltiples factores; asimismo, es ideal el acompańamiento de otras instituciones, para generar cadenas de sostenibilidad y fomentar la circularidad en más sectores productivos. 


\section{Recomendaciones y conclusiones}

Al ser este capítulo un estudio meramente teórico, se recomienda contar con la disposición de las autoridades del Ejército Nacional de Colombia para realizar una debida investigación de flujos de energía y materia, al igual que un análisis contable de su cadena de suministro y un enlace con la comunidad militar, elementos claves para implementar las debidas metodologías y desarrollar nuevos modelos de transformación hacia la circularidad.

Igualmente, al momento de llegar a posibles iniciativas basadas en estas investigaciones, es necesario priorizar resultados, tener en cuenta su inversión, viabilidad, impacto global y contribución a la comunidad; de esta manera todo proyecto será duplicable en otras instituciones e instancias.

En cuanto a la transformación de sistemas, es importante lograr una cohesión de actores y acciones de los diferentes sectores productivos, de manera que se logre una articulación de las cadenas de suministro, y desde el primer actor hasta el posconsumo se logre cerrar estos ciclos y fomentar la expansión de este desarrollo al resto de sectores del país.

Lograr dirigir a instituciones de alto valor, como el Ejército Nacional de Colombia, por el camino del desarrollo sostenible, no solo demuestra el compromiso de esta institución por el país, sino también su posicionamiento como un medio para afianzar este cambio de modelos en Colombia. Si desde todas sus escuelas y dependencias se logra la medición y definición de indicadores, la formulación de alternativas, la generación de capacidades y el seguimiento de estos resultados, entonces, desde las industrias y los hogares colombianos también se puede lograr.

La situación actual del orbe no solo es el reflejo de un mal sistema mundial, sino la respuesta del mismo planeta que urge por un cambio. Como ha sucedido en el pasado, toda crisis viene acompañada de un renacimiento, esperemos que la economía circular y el desarrollo sostenible hagan parte de las soluciones y esperanzas para nuestra sociedad.

\section{Referencias}

Andrews, D. (2015). The Circular Economy, Design Thinking and Education for Sustainability. Lepu Local Economy Policy Unit, 30(3), 305-315.

Banco Mundial. (2016). What a Waste 2.0: Trends in Solid Waste Management. https://datatopics.worldbank.org/what-a-waste/trends_in_solid_waste_management.html 
Bonciu, F. (2014). The European Economy: From Linear to Circular Economy. Romanian Journal of European Affairs, 14(4), 78-91.

Boulding, K. (1966). The economics of the coming spaceship Earth. In H. Jarrett (Ed), Environmental quality in a growing economy. Johns Hopkins University Press.

Comando General Fuerzas Militares de Colombia. (2015). Plan Estratégico Militar —PEM 2030-. Comando General Fuerzas Militares de Colombia. https://www.dipor.co/\%7CDoctrina\%20 Publica\%7C/1\%20Comando\%20General\%20Fuerzas\%20Militares/Manuales/PEM\%20 2030\%20FINAL\%20OBJETIVOS\%20ESTRAT\%C3\%89GICOS.pdf

Cuervo, L., \& Padilla, J. (s. f.). Cambio de fuente de abastecimiento de agua para procesos secundarios. Productos Ramo S. A. http://www.redescar.org/sites/default/files/2019-11/4.Ficha-proyectoPMl-Ramo1.pdf

Departamento Nacional de Planeación - DNP. (2018). Política de Crecimiento Verde. Departamento Nacional de Planeación. Tomado de: https://www.dnp.gov.co/Crecimiento-Verde/Paginas/ Politica-crecimiento-verde.aspx

Edirisinghe, E., \& Bogahawatte, S. (2018). Sustainable Water Supply Management System for Panagoda Army Cantonment. http://ir.kdu.ac.lk/handle/345/2562

Ejército Nacional de Colombia (s. f.). Principios. Ejército Nacional de Colombia. https://www.ejercito. mil.co/conozcanos/principios_valores/principios

Ejército Nacional de Colombia. (s. f.). Visión. Ejército Nacional de Colombia. https://www.ejercito. mil.co/conozcanos/mision_vision/vision

Ellen MacArthur Foundation. (2015). Hacia una economía circular: motivos económicos para una transición acelerada. https:/www.ellenmacarthurfoundation.org/assets/downloads/publications/ Executive_summary_SP.pdf

Geisendorf, S., \& Pietrulla, F. (2017). The circular economy and circular economic concepts-a literature analysis and redefinition. Thunderbird International Business Review, 60(5), 771-782.

Glenn, J., Gordon, T., \& Perelet, R. (1998). Defining environmental security: Implications for the US army. Army Environmental Policy Institute.

Global Footprint Network \& Earth Overshoot Day. (2020). El Día del Sobregiro de la Tierra es el 22 de agosto, más de tres semanas más tarde que el año pasado. Earth Overshoot Day. https://www. overshootday.org/newsroom/press-release-june-2020-spanish/

Jawahir, I., \& Bradley, R. (2016). Technological Elements of Circular Economy and The Principles of 6R-Based Closed-Loop Material Flow in Sustainable Manufacturing. Procedia CIRP, 40(1), 103-108.

Joustra, D., De Jong, E., \& Engelaer, F. (2013). Guided Choices: Towards a Circular Business Model. C2C BIZZ.

Keeble, B. (1988). The Brundtland report: 'Our common future'. Medicine and war, 4(1), 17-25.

Kuperman, R., Checkai, R., Simini, M., Phillips, C., Kolakowski, J., \& Chester, N. (2006). Ecological Soil Screening Levels for Invertebrates at Explosives-Contaminated Sites: Supporting Sustainability of Army Testing and Training. Edgewood Chemical Biological Center Aberdeen Proving Ground Md. https://apps.dtic.mil/dtic/tr/fulltext/u2/a481509.pdf 
Kwon, H., \& Park, H. (2019). Creating Sustainable and Climate Shared Value in Public Institution: Lessons from a Case of Korea Army Cadet Military School. Sustainability, 11(14), 37-96.

Lachman, B., Pint, E., Cecchine, G., \& Colloton, K. (2009). Developing Headquarters Guidance for Army Installation Sustainability Plans in 2007. RAND Corporation.

Lacy, P., \& Rutqvist, J. (2016). Waste to Wealth: The Circular Economy Advantage. Springer.

Meadows, D., Meadows, D., Randers, J., \& Behrens, W. (1972). The limits to growth. New York, 102(1972), 27.

Ministerio de Ambiente y Desarrollo Sostenible, Ministerio de Comercio, Industria y Turismo, y Gobierno Nacional de Colombia. (2019). Estrategia nacional de economía circular: Cierre de ciclos de materiales, innovación tecnológica, colaboración y nuevos modelos de negocio. Presidencia de la República.

Presidencia de la República. (2019). Con la puesta en marcha de la Campaña 'Artemisa', buscamos parar la hemorragia deforestadora que se ha visto en los últimos años en el país: Presidente Duque. Presidencia de la República. https://id.presidencia.gov.co/Paginas/prensa/2019/190428-puesta-marcha-Campana-Artemisa-buscamos-parar-hemorragia-deforestadora-ha-visto-ultimos-anios-pais-Duque.aspx

Rahman, M. (2019). Capability enhancement of Bangladesh Army to perform environmental protection and refugee control (Master dissertation). US Army Command and General Staff College, Fort Leavenworth United States.

RedES-CAR. (s. f., a). La iniciativa: Alianza por el cambio. http://www.redescar.org/la-iniciativa-redescar/alianza-por-el-cambio

RedES-CAR. (s. f., b). La iniciativa: Metodología. http://www.redescar.org/la-iniciativa-redescar/ metodologia

RedES-CAR. (s. f., c). Resultado: Centro de Conocimiento de Casos. http://www.redescar.org/resultados-redescar/casos-de-exito

Restrepo, C. (2014). Propuesta para el mejoramiento de la cadena de suministros y abastecimiento del Ejercito Nacional de Colombia, para responder a las necesidades del siglo XXI (Tesis de maestría). Universidad Militar Nueva Granada. https://repository.unimilitar.edu.co/handle/10654/13007

Rozo, G. (2019). Estado del Arte de la Economía Circular en Colombia (Tesis de pregrado). Universidad Cooperativa de Colombia.

Sariatli, F. (2017). Linear Economy Versus Circular Economy: A Comparative and Analyzer Study for Optimization of Economy for Sustainability. Visegrad Journal on Bioeconomy and Sustainable Development, 6(1), 31-34.

Stahel, W. (1982). Product-life factor (Mitchell Prize Winning Paper 1982). The Product-Life Institute. http://www.product-life.org/en/major-publications/the-product-life-factor

Suárez-Eiroa, B., Fernández, E., Méndez-Martínez, G., \& Soto-Oñate, D. (2019). Operational principles of Circular Economy for Sustainable Development: Linking theory and practice. Journal of Cleaner Production, 214, 952-961.

Tovar, W. (2020). Gestión Ambiental en el Ejército Nacional. En C. Bentacour, \& J. Cardona (Dirc.), Memorias Primer Encuentro de Desarrollo y Sostenibilidad Ambiental, pp. 9-11. Escuela de Armas Combinadas del Ejército - Escuela de Aviación del Ejército. 
148 Miradas de innovación, sostenibilidad y desarrollo en torno a la gestión ambiental en el Ejército Nacional de Colombia

United Nations, Department of Economic and Social Affairs \& Population Division. (2019). World population prospects 2019: Highlights. United Nations Department for Economic and Social Affairs.

Van Hoof, B., Hernández, J., Samper, H., \& Saker, A. (2018). Liderazgo Ambiental para la transformación productiva: lecciones de América Latina. Editorial Alfaomega.

Warnock, D. (2008). Institutionalizing Sustainability into the Total Army. U. S. Army War College, Carlisle Barracks 